\title{
ANÁLISIS CINEMÁTICO Y DISEÑO DE UN MECANISMO DE CUATRO BARRAS PARA FALANGE PROXIMAL DE DEDO ANTROPOMÓRFICO
}

\author{
KYNEMATIC ANALYSIS AND DESIGN OF A FOUR-BAR MECHANISM FOR \\ PROXIMAL FINGER PHALANX OF ANTHROPOMORPHIC FINGER
}

\author{
Édgar Alfredo Portilla Flores
}

Ph.D. Ing. Electrónico, Docente del Centro de Innovación y Desarrollo Tecnológico en Cómputo - Instituto Politécnico Nacional, Departamento de Posgrado, Área de Mecatrónica, México, D.F., 07700, México. aportilla@ipn.mx

Oscar Fernando Avilés Sánchez

Ph.D. Ing. Electrónico, Docente de la Universidad Militar Nueva Granada, Programa de Ingeniería Mecatrónica, Grupo de Investigación en Mecatrónica DAVINCI, Bogotá D. C., Colombia. oscar.aviles@ unimilitar.edu.co

\section{Roberto Piña Quintero}

Centro de Innovación y Desarrollo Tecnológico en Cómputo -Instituto Politécnico Nacional, Departamento de Posgrado, Área de Mecatrónica, México, D.F., 07700, México, rpinaq0800@ipn.mx

\section{Paola Andrea Niño Suárez}

Ph.D. Ing. Electrónico, Docente de la Universidad Militar Nueva Granada, Programa de Ingeniería Mecatrónica, Grupo de Investigación en Mecatrónica DAVINCI, Bogotá D. C., Colombia. paola.nino@ unimilitar.edu.co

\section{Edgar Moya Sánchez}

Centro de Innovación y Desarrollo Tecnológico en Cómputo -Instituto Politécnico Nacional, Departamento de Posgrado, Área de Mecatrónica, México, D.F., 07700, México.

\section{María Aurora Molina Vilchis}

M. en C. Centro de Innovación y Desarrollo Tecnológico en Cómputo -Instituto Politécnico Nacional, Departamento de Posgrado, Área de Mecatrónica, México, D.F., 07700, México. mamolinav@ipn.mx

Fecha de recepción: 2 de diciembre de 2009

Fecha de aprobación: 13 de abril de 2010

\section{RESUMEN}

En este artículo, se presenta el análisis cinemático y una metodología de diseño paramétrico de la falange proximal para un dedo antropomórfico ${ }^{1}$ de un grado de

1 Antropomorfismo: dar características humanas a cosas o mecanismos que no lo son. 
libertad. El diseño del mecanismo se lleva a cabo, tomando diez posiciones de la barra de salida del mismo. Se presenta la simulación dinámica, utilizando un modelo de CAD para comprobar la factibilidad de los resultados obtenidos por el mecanismo. Por último, se muestra el análisis comparativo entre los resultados de la cinemática obtenidos numéricamente y los experimentales.

Palabras clave: mecanismo de cuatro barras, dedo antropomórfico, diseño paramé-trico.

\begin{abstract}
This paper shows the kinematic analysis and a parametric design methodology of the proximal phalanx for an anthropomorphic finger using a one degree-of-freedom. The mechanism design is done by taking ten positions from its output bar. A dynamic simulation is given using a CAD model to verify the feasibility of the mechanism results. Finally, we show the comparative analysis between kinematics numerically obtained and other experimental results.
\end{abstract}

Keywords: four-bar mechanism, anthropomorphic finger, parametric design.

\title{
INTRODUCCIÓN
}

El éxito que ha tenido el hombre en su evolución, se debe a la increíble habilidad que posee para explorar y estudiar el mundo que lo rodea. Esta tarea se debe en gran parte, a la capacidad de manipulación del ser humano. Con la mano, se puede agarrar, sostener y manipular objetos con gran destreza, haciendo de ella, una herramienta importante en el desempeño de las tareas del diario vivir.

El estudio del agarre humano como modelo inspirador para el diseño de manos robóticas, ha llevado a la construcción de innovadores pero costosos prototipos de dedos, para utilizarse como partes funcionales de robots antropomórficos. Ejemplos de referencia pueden ser los dedos utilizados en las manos de Stanford/JPL Hand [2], Utah/MIT Hand [3], SPRING Hand [4], DLR Hand [5], UNT Hand [6], UMNG Hand [7]. Otros trabajos relacionados con el desarrollo de dedos son el de M. Cecacarrlli et al [8], que propusieron la cinemática del movimiento de dedos humanos, usando secuencias de grabación de video y foto como una metodología para determinar la función del movimiento. F. García et al [9], utilizaron un sistema de actuación que emula el sistema muscular del dedo, representado por un sistema de transmisión basado por tendones. Fabrizio Lotti et al [10], desarrollaron un dedo que consta de dos grados de libertad, de bajo peso, que utiliza eslabones rígidos unidos con elementos flexibles; y los actuadores empleados son de desplazamiento lineal. En la tabla 1, se presenta una revisión de algunos trabajos relacionados con manos robóticas. 
Tabla 1. Revisión Manos robóticas

\begin{tabular}{|c|c|c|c|c|c|}
\hline Nombre Mano & Autor & Año & $\begin{array}{c}\text { Tamaño } \\
\text { (norm.) }\end{array}$ & $\begin{array}{c}\text { \# de } \\
\text { Dedos }\end{array}$ & GDL \\
\hline Humana & (Kandel) & 2000 & 1 & 5 & 22 \\
\hline Soft Gripper & (Hirose) & 1976 & $>1$ & 2 & 10 \\
\hline Okada & (Okada) & 1982 & $>1$ & 3 & 11 \\
\hline Stanford/JPL & (Salisbury) & 1982 & $\sim 1,2$ & 3 & 9 \\
\hline Utah/MIT & (Jacobsen) & 1986 & $>2$ & 4 & 16 \\
\hline Barret & (Townsend) & 1988 & $\sim 1$ & 3 & 4 \\
\hline Belgrade/USC & (Bekey) & 1989 & $\sim 1,1$ & 5 & 6 \\
\hline UB & (Melchiori) & 1992 & $\sim 1$ & 3 & 13 \\
\hline MARCUS & (Kyberd) & 1995 & $>1,1$ & 3 & 2 \\
\hline NTU & (Li Ren) & 1996 & $\sim 1$ & 5 & 17 \\
\hline MARS & (Gosselin) & 1996 & $>1$ & 3 & 12 \\
\hline DLR I & (Butterfas) & 1997 & $>1$ & 4 & 12 \\
\hline AMADEUS & (Lane) & 1997 & $>1$ & 3 & n.a \\
\hline DIST & (Caffaz) & 1998 & $>1$ & 5 & 16 \\
\hline Hokkaido & (Fujii) & 1998 & $>1$ & 5 & 7 \\
\hline ASI Gripper & (Melchiorri) & 1998 & $>1$ & 3 & 3 \\
\hline DLR I & (Liu - Buterfass) & 1998 & 1,5 & 4 & 12 \\
\hline Goldfinger & (Ramos) & 1999 & $>1$ & 4 & 12 \\
\hline Robonaut & (Lovchik) & 1999 & $\sim 1,5$ & 5 & $12+2$ \\
\hline Tokyo & (Lee) & 1999 & $» 1$ & 5 & 12 \\
\hline Ultralight & (Schultz) & 2000 & $>1$ & 5 & 13 \\
\hline Southampton & (Light) & 2000 & $>1$ & 5 & 6 \\
\hline SARAH & (Lalibeerte) & 2000 & $>1$ & 3 & 10 \\
\hline LMS & (Gazeau) & 2001 & $\sim 1$ & 4 & 16 \\
\hline Karlsruhe & (Schulz) & 2001 & $\sim 1$ & 5 & 17 \\
\hline DLR II & (Liu Butterfass) & 2001 & 1,5 & 4 & 13 \\
\hline Gifu & (Kawasaki) & 2001 & $\| 1$ & 5 & 16 \\
\hline Ottobock SUVA & (Otto Bock) & 2002 & $\sim 1$ & 3 & 1 \\
\hline RTR Hand 1 & (Massa) & 2002 & $» 1$ & 3 & 6 \\
\hline RTR Hand 2 & (Massa) & 2002 & $» 1$ & 3 & 9 \\
\hline Shadow & (Shadow Co) & 2002 & »1,1 & 5 & 24 \\
\hline SPRING Hand & (Carroza) & 2004 & $\sim 1,5$ & 3 & 8 \\
\hline UMNG & (Ocampo) & 2004 & $\sim 1$ & 4 & $12+2$ \\
\hline
\end{tabular}

Los sistemas robóticos avanzados están pasando del concepto clásico de estructuras precisas y rígidas, muchas veces pesadas y complejas, a estructuras más flexibles y livianas, con la perspectiva de un aumento en su desempeño, alta simplicidad mecánica y en consecuencia, con una considerable reducción de costos. Los robots, en especial los humanoides y manipuladores industriales, han sido proyectados para ayudar en diversos trabajos en la industria y en el hogar. Por lo tanto, estos robots necesitan tener la capacidad de realizar las tareas que normalmente hacen los humanos, y los mismos deben ser dotados de una herramienta terminal que pueda manipular objetos con la misma destreza y capacidad de un ser humano. 
La finalidad del mecanismo descrito en este artículo (mecanismo de cuatro barras), es construir la falange proximal de un dedo antropomórfico cuyo funcionamiento sea similar al de su homólogo humano. La mano humana está conformada por una palma central (metacarpo), de donde surgen cinco dedos, está unida al antebrazo por una unión llamada muñeca (carpo). Además, la mano está compuesta de varios músculos y ligamentos diferentes que permiten una gran cantidad de movimientos y significativa destreza.

La mano humana, Instrumento de Instrumentos, deriva su agilidad de tres funciones esenciales:

1. Pronación y supinación: son los movimientos del antebrazo que tienen por efecto, hacer que la mano ejecute una rotación de fuera hacia adentro, y el movimiento del antebrazo que coloca la mano con la palma hacia arriba y el pulgar hacia fuera respectivamente. Estos movimientos adaptan la mano a cualquier circunstancia.

2. Flexión y cierre de los dedos: es una función muy importante, por que gracias a ella es posible realizar una gran variedad de tareas.

3. Oposición del dedo pulgar: situado al frente de la palma y los otros dedos, el pulgar puede usarse para el desarrollo de grippers multidedos, particularmente con el dedo índice.

El conocimiento anatómico de la mano humana para el diseño de efectores finales tipo manos multidedos, es un paso indispensable. Para un conocimiento anatómico detallado, están los trabajos desarrollados por Tubiana [14] y Caffiniére [15], los cuales hacen un análisis interesante de ciertos aspectos de la fisiología de la mano, con el fin de extraer las características mecánicas más determinantes para poder realizar así, un modelo correcto.

La mano humana es una estructura compleja que tiene 21 grados de libertad. Los dedos tienen tres falanges y cuatro grados de libertad; dos de ellos en su articulación con la palma (llamada metacarpo- falangeal, ya que articula la falange proximal con el hueso metacarpiano) y los otros dos del movimiento entre la falange media y distal. Y cinco grados de libertad para el pulgar que tiene dos falanges y un metacarpo [1]. La figura 1 muestra la fisiología de la mano. 

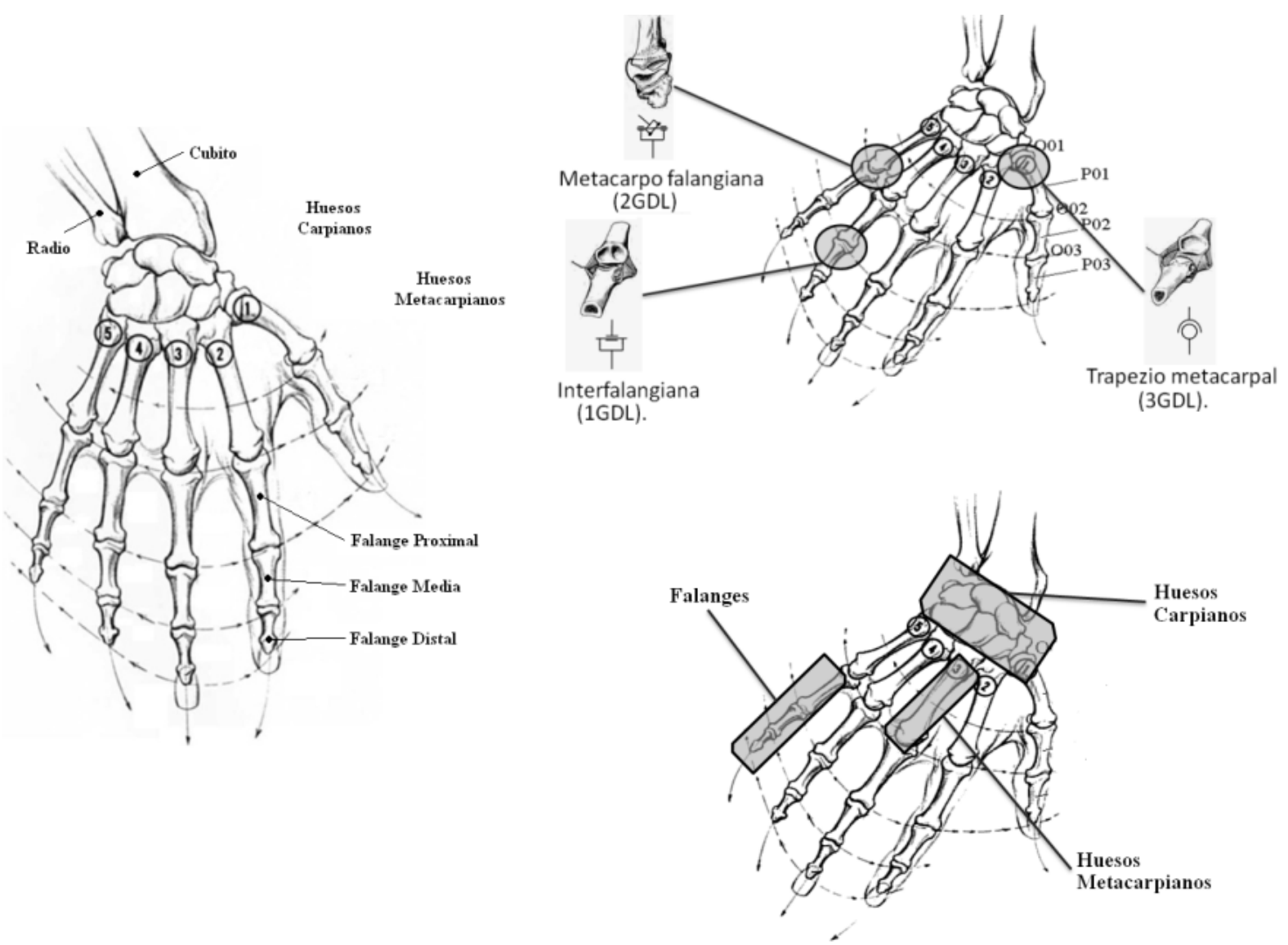

Figura 1. Fisiología de una mano humana

Las manos robóticas requieren un trabajo especial de diseño debido a su capacidad de operación: la flexión de todos los dedos y la movilidad del pulgar. Las soluciones no son fáciles $\mathrm{y}$, por lo general, requieren de sistemas especiales de engranajes, o actuadores poco comunes, muy miniaturizados, y diversos tipos de implementaciones para los tendones artificiales.

El uso principal de las manos es el de tomar y sostener objetos, aunque de estos usos generales derivan muchos más, debido a la gran versatilidad de movimiento del que es capaz la mano, así como por la precisión que puede alcanzar en estos movimientos, [14, 15]. Para simular mecánicamente estas funciones, se utiliza, por ejemplo, mecanismos de cuatro barras para el desarrollo de dedos robóticos. Si se asume que todos los dedos, excepto el pulgar, son similares en su funcionamiento se podría reproducir los dedos necesarios para la construcción de una mano robótica. 


\section{DESCRIPCIÓN DEL DEDO ANTROPOMÓRFICO}

En Avilés et al [11], se presenta el diseño y construcción de un dedo robótico, cuya entrada es un tornillo sin fin, para conversión de movimiento rotacional en lineal; así mismo, se construye el dedo a partir del modelo obtenido de las simulaciones en CAD. Se lleva a cabo, mediciones de posición en el prototipo construido, las cuales se comparan con las obtenidas mediante simulación en CAD. El mecanismo actuador está integrado por dos elementos cruzados de cuatro barras acoplados entre ellos (figura 2a), donde la falange distal, media y proximal se denominan F3, F2 y F1 respectivamente. En la figura $2 \mathrm{~b}$ se puede observar el mecanismo actuador representado mediante pares giratorios $(\text { clase } V)^{2}$. El eslabón de entrada AB (clase IV), se desplaza en forma lineal, provocando el movimiento del mecanismo DHIEJ mediante el par rotacional C; así mismo el par rotacional $\mathrm{K}$ activa el segundo mecanismo GFML, con el fin de describir la trayectoria de un dedo de la mano humana.

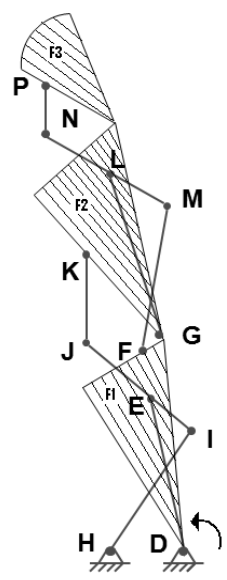

Figura 2a. Mecanismo del dedo índice.

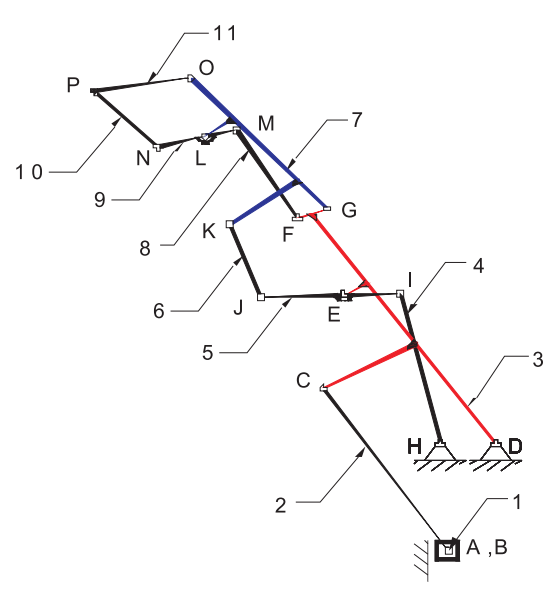

Figura 2b. Mecanismo analítico del dedo índice.

En este artículo se presentará el mecanismo cruzado de cuatro barras que se observa en la figura 3, y que equivale a la falange proximal F1, del dedo, donde HD es el eslabón fijo, DE es el eslabón de entrada, IE el eslabón acoplador y HI es el eslabón de salida, figura 3 , el análisis de la falange media y distal se realiza de la misma forma, pues son una extensión del mecanismo que aquí se presenta.

Todos los pares cinemáticos se dividen en clases de acuerdo con el número de condiciones de enlace, impuestas por ellas al movimiento relativo de sus eslabones, ya que el número de condiciones de enlace puede ser de 1 a 5, entonces por correspondencia se tienen pares cinemáticos de I, II, III, IV y V clases. 

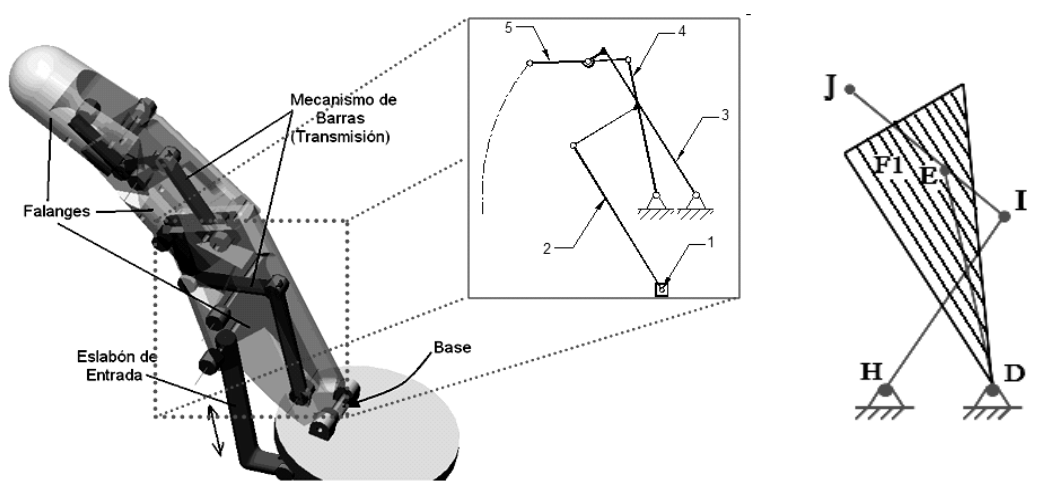

Figura 3. Mecanismo de la falange proximal del dedo índice [11]

\section{DISEÑO DEL MECANISMO DE CUATRO BARRAS}

Considérese el mecanismo cruzado de cuatro barras de la figura 3 , representado vectorialmente en la figura 4 . Como se puede observar, este mecanismo forma una cadena cinemática cerrada. Con el propósito de hacer el análisis de posición, se consideró el ángulo de entrada $\theta_{3}$, tomando al eslabón $r_{4}$ fijo.

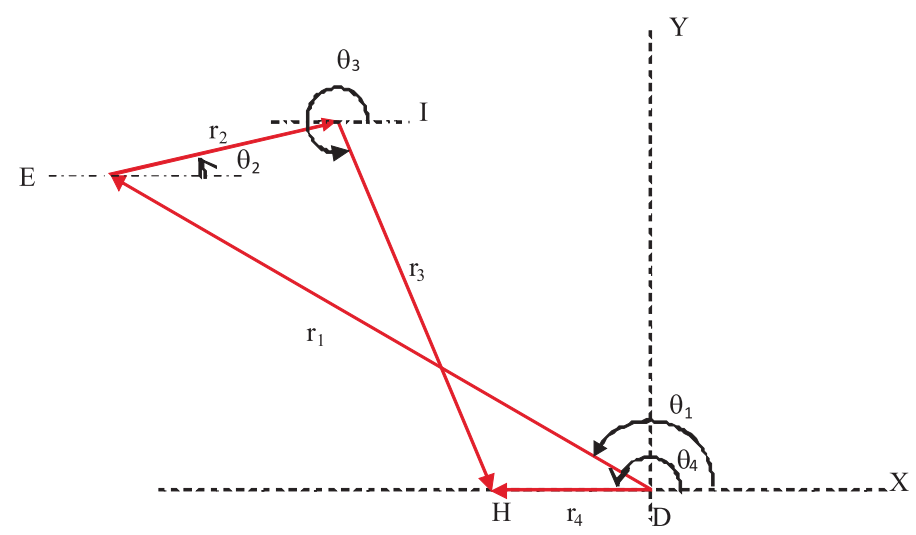

Figura 4. Mecanismo cruzado de cuatro barras

Nota 2 (Cont.)

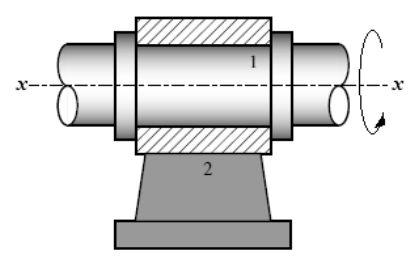

Por ejemplo: en la figura, se muestra un par de $\mathrm{V}$ clase; cada uno de los eslabones de esta junta posee sólo un movimiento simple: giro alrededor del eje $\mathrm{x}-\mathrm{x}$. Por esto, el número de grados de libertad $\mathrm{H}$ de este par, es igual a uno y el número de condiciones de enlace:

$$
S=6-H=6-1=5 \text {. }
$$

Donde $\mathrm{H}$ representa el número de grados de libertad y $\mathrm{S}$ el número de condiciones de enlace $(1 \leq S \leq 5)$. Este par recibe el nombre de par o junta giratoria. 
Sea la ecuación de cierre de mecanismo o de circuito siguiente:

$$
\vec{r}_{1}+\vec{r}_{2}+\vec{r}_{3}-\vec{r}_{4}=0
$$

Expresada en términos de los componentes $\mathrm{X}$ e $\mathrm{Y}, \mathrm{y}$ considerando que el mecanismo debe cumplir con las posiciones deseadas, se tiene entonces que:

Componentes en X:

$$
r_{1} \cos \theta_{1 i}+r_{2} \cos \theta_{2 i}+r_{3} \cos \theta_{3 i}-r_{4} \cos \theta_{4}=0
$$

Componentes en Y:

$$
r_{1} \operatorname{sen} \theta_{1 i}+r_{2} \operatorname{sen} \theta_{2 i}+r_{3} \operatorname{sen} \theta_{3 i}-r_{4} \operatorname{sen} \theta_{4}=0
$$

Donde el subíndice $i$ corresponde a la i-esíma posición de precisión que debe cumplir el mecanismo. Por otra parte, de la figura 4 se observa que al ser fijo el eslabón $r_{4}$, su ángulo es constante y de valor conocido, es decir $\theta_{4}=\pi$. Por lo tanto, se obtiene:

$$
\begin{gathered}
r_{1} \cos \theta_{1 i}+r_{2} \cos \theta_{2 i}+r_{3} \cos \theta_{3 i}+r_{4}=0 \\
r_{1} \operatorname{sen} \theta_{1 i}+r_{2} \operatorname{sen} \theta_{2 i}+r_{3} \operatorname{sen} \theta_{3 i}=0
\end{gathered}
$$

Para encontrar la relación entre el ángulo de entrada $\theta_{3}$ y el ángulo de salida $\theta_{1}$, se debe eliminar el ángulo del eslabón acoplador $\theta_{2}$.

$$
\begin{gathered}
r_{2} \cos \theta_{2 i}=-r_{1} \cos \theta_{1 i}-r_{3} \cos \theta_{3 i}-r_{4} \\
r_{2} \operatorname{sen} \theta_{2 i}=-r_{1} \operatorname{sen} \theta_{1 i}-r_{3} \operatorname{sen} \theta_{3 i}
\end{gathered}
$$

Elevando al cuadrado ambos términos de la igualdad y sumando ambas ecuaciones se tiene que:

$$
\begin{gathered}
r_{2}^{2} \cos ^{2} \theta_{2 i}+r_{2}^{2} \operatorname{sen}^{2} \theta_{2 i}=\left(-r_{1} \cos \theta_{1 i}-r_{3} \cos \theta_{3 i}-r_{4}\right)^{2}+\left(-r_{1} \operatorname{sen} \theta_{1 i}-r_{3} \operatorname{sen} \theta_{3 i}\right)^{2} \\
r_{2}^{2}=r_{1}^{2}+r_{3}^{2}+r_{4}^{2}+2 r_{1} r_{3} \cos \left(\theta_{1 i}-\theta_{3 i}\right)+2 r_{1} r_{4} \cos \theta_{1 i}+2 r_{3} r_{4} \cos \theta_{3 i}
\end{gathered}
$$

Deja en evidencia el término que involucra las variables de entrada y de salida:

$$
\cos \left(\theta_{1 i}-\theta_{3 i}\right)=-\frac{r_{4}}{r_{3}} \cos \theta_{1 i}-\frac{r_{4}}{r_{1}} \cos \theta_{3 i}+\frac{r_{2}^{2}-r_{1}^{2}-r_{3}^{2}-r_{4}^{2}}{2 r_{1} r_{3}}
$$

Definiendo las variables $k_{1}, k_{2}$ y $k_{3}$ 


$$
k_{1}=-\frac{r_{4}}{r_{3}}, \quad k_{2}=-\frac{r_{4}}{r_{1}}, \quad k_{3}=\frac{r_{2}-r_{1}-r_{3}-r_{4}}{2 r_{1} r_{3}}
$$

La ecuación (10) se convierte en

$$
\cos \left(\theta_{1 i}-\theta_{3 i}\right)=k_{1} \cos \theta_{1 i}+k_{2} \cos \theta_{3 i}+k_{3}
$$

La cual se puede reescribir de la siguiente manera:

$$
\cos \left(\theta_{1 i}-\theta_{3 i}\right)-k_{1} \cos \theta_{1 i}-k_{2} \cos \theta_{3 i}-k_{3}=0
$$

Siendo (13) la ecuación de Freudenstein [12].

\section{MINIMIZACIÓN DEL ERROR EN LAS POSICIONES}

Con el propósito de obtener un mínimo de error, en las posiciones que debe cumplir el mecanismo actuador, se aplica el método de mínimos cuadrados. Sea la función $D$, definida en términos de la ecuación que establece las posiciones del mecanismo actuador, es decir:

$$
D=\sum_{i=1}^{n}\left[\cos \left(\theta_{1 i}-\theta_{3 i}\right)-k_{1} \cos \theta_{1 i}-k_{2} \cos \theta_{3 i}-k_{3}\right]^{2}
$$

Tomando en cuenta, que se debe minimizar el error en las posiciones que debe cumplir el mecanismo actuador y observando la dependencia matemática de la función $D$ de los parámetros $k$ 's definidos en la ecuación (11). Se debe cumplir para obtener el menor error entre los ángulos deseados:

$$
\frac{\partial D}{\partial k_{1}}=0, \quad \frac{\partial D}{\partial k_{2}}=0, \quad \frac{\partial D}{\partial k_{3}}=0
$$

Desarrollando cada una de las derivadas parciales, se obtiene:

$$
\begin{aligned}
& \frac{\partial D}{\partial k_{1}}=2 k_{1} \sum_{i=1}^{n} \cos ^{2} \theta_{1 i}+2 k_{2} \sum_{i=1}^{n} \cos \theta_{1 i} \cos \theta_{3 i}+2 k_{3} \sum_{i=1}^{n} \cos \theta_{1 i}-2 \sum_{i=1}^{n} \cos \theta_{1 i} \cos \left(\theta_{1 i}-\theta_{3 i}\right)=0 \\
& \frac{\partial D}{\partial k_{2}}=2 k_{2} \sum_{i=1}^{n} \cos ^{2} \theta_{3 i}+2 k_{1} \sum_{i=1}^{n} \cos \theta_{1 i} \cos \theta_{3 i}+2 k_{3} \sum_{i=1}^{n} \cos \theta_{3 i}-2 \sum_{i=1}^{n} \cos \theta_{3 i} \cos \left(\theta_{1 i}-\theta_{3 i}\right)=0 \\
& \frac{\partial D}{\partial k_{3}}=2 n k_{3}+2 k_{1} \sum_{i=1}^{n} \cos \theta_{1 i}+2 k_{2} \sum_{i=1}^{n} \cos \theta_{3 i}-2 \sum_{i=1}^{n} \cos \left(\theta_{1 i}-\theta_{3 i}\right)=0
\end{aligned}
$$


Expresando las ecuaciones (16), en forma matricial, se obtiene:

$$
\left[\begin{array}{ccc}
\sum_{i=1}^{n} \cos ^{2} \theta_{1 i} & \sum_{i=1}^{n} \cos \theta_{1 i} \cos \theta_{3 i} & \sum_{i=1}^{n} \cos \theta_{1 i} \\
\sum_{i=1}^{n} \cos \theta_{1 i} \cos \theta_{3 i} & \sum_{i=1}^{n} \cos ^{2} \theta_{3 i} & \sum_{i=1}^{n} \cos \theta_{3 i} \\
\sum_{i=1}^{n} \cos \theta_{1 i} & \sum_{i=1}^{n} \cos \theta_{3 i} & n
\end{array}\right]\left[\begin{array}{l}
k_{1} \\
k_{2} \\
k_{3}
\end{array}\right]=\left[\begin{array}{c}
\sum_{i=1}^{n} \cos \theta_{1 i} \cos \left(\theta_{1 i}-\theta_{3 i}\right) \\
\sum_{i=1}^{n} \cos \theta_{3 i} \cos \left(\theta_{1 i}-\theta_{3 i}\right) \\
\sum_{i=1}^{n} \cos \left(\theta_{1 i}-\theta_{3 i}\right)
\end{array}\right]
$$

Donde los coeficientes $k_{1}, k_{2}$ y $k_{3}$ son los coeficientes de la ecuación de Freudenstein. Al resolver el sistema de ecuaciones dado por (17), y proponiendo un valor para el eslabón fijo $r_{4}$ se pueden determinar la longitud de los demás eslabones.

\section{RESULTADOS NUMÉRICOS}

Con el propósito de obtener el diseño del mecanismo actuador, se utilizó MATLAB ${ }^{\circledR}$ para el cálculo requerido. En la tabla 2, se muestran las 10 posiciones utilizadas para determinar las dimensiones del mecanismo.

Tabla 2. Posiciones del mecanismo.

\begin{tabular}{|c|c|c|c|c|c|c|c|c|}
\hline \multirow{2}{*}{ Posición } & \multicolumn{2}{|c}{ Punto E } & \multicolumn{2}{c|}{ Punto I } & \multicolumn{2}{c|}{ Punto H } & \multicolumn{2}{c|}{ Punto D } \\
\cline { 2 - 8 } & $X$ & $Y$ & $X$ & $Y$ & $X$ & $Y$ & $X$ & $Y$ \\
\hline 1 & -18.9749 & 24.0306 & -11.9795 & 24.2845 & -6.9989 & 0.0000 & 0.0000 & 0.0000 \\
\hline 2 & -21.5732 & 21.7281 & -14.8085 & 23.5277 & -6.9989 & 0.0000 & 0.0000 & 0.0000 \\
\hline 3 & -23.8943 & 19.1463 & -17.6810 & 22.3704 & -6.9989 & 0.0000 & 0.0000 & 0.0000 \\
\hline 4 & -25.9081 & 16.3183 & -20.5152 & 20.7811 & -6.9989 & 0.0000 & 0.0000 & 0.0000 \\
\hline 5 & -27.5888 & 13.2805 & -23.2178 & 18.7481 & -6.9989 & 0.0000 & 0.0000 & 0.0000 \\
\hline 6 & -28.6413 & 10.8254 & -25.1450 & 16.8897 & -6.9989 & 0.0000 & 0.0000 & 0.0000 \\
\hline 7 & -29.4758 & 8.2880 & -26.8912 & 24.7933 & -6.9989 & 0.0000 & 0.0000 & 0.0000 \\
\hline 8 & -30.0860 & 5.6875 & -28.4154 & 12.4852 & -6.9989 & 0.0000 & 0.0000 & 0.0000 \\
\hline 9 & -30.4672 & 3.0437 & -29.6827 & 9.9995 & -6.9989 & 0.0000 & 0.0000 & 0.0000 \\
\hline 10 & -30.6166 & 0.3767 & -30.6660 & 7.3765 & -6.9989 & 0.0000 & 0.0000 & 0.0000 \\
\hline
\end{tabular}


Debido a que la ecuación (17), es función de los ángulos de entrada y salida del mecanismo actuador, se llevó a cabo el cálculo de dichos ángulos mediante el Toolbox de minimización restringida de MATLAB ${ }^{\circledR}$ para cada posición del mecanismo. La tabla 3 , muestra los valores de los ángulos para cada una de las posiciones que debe cumplir el mecanismo actuador.

Tabla 3. Ángulos para las posiciones

\begin{tabular}{|c|c|}
\hline $\begin{array}{c}\text { Ángulo de entrada } \theta_{3} \\
\text { (rad) }\end{array}$ & $\begin{array}{c}\text { Ángulo de salida } \theta_{1} \\
\text { (rad) }\end{array}$ \\
\hline 3.9357 & 1.0400 \\
\hline 4.1277 & 1.2574 \\
\hline 4.3197 & 1.5410 \\
\hline 4.5117 & 1.7736 \\
\hline 4.7037 & 2.0165 \\
\hline 4.8956 & 2.2205 \\
\hline 5.0876 & 2.4094 \\
\hline 5.2796 & 2.5722 \\
\hline 5.4716 & 2.7305 \\
\hline 5.6636 & 2.8828 \\
\hline 5.8556 & 3.0331 \\
\hline 6.0476 & 3.1822 \\
\hline 6.2396 & 3.3298 \\
\hline
\end{tabular}

A partir de los datos de la tabla 3, se resolvió el sistema de ecuaciones simultáneas establecido por la ecuación 17, al resolver dicho sistema, las dimensiones obtenidas de los eslabones del mecanismo actuador, se muestran en la tabla 4.

Tabla 4. Dimensiones del mecanismo

\begin{tabular}{|c|c|}
\hline Eslabón & $\begin{array}{c}\text { Longitud } \\
(\mathrm{mm})\end{array}$ \\
\hline$r 1$ & 30.6130 \\
\hline$r 2$ & 6.9973 \\
\hline$r 3$ & 24.7825 \\
\hline$r 4$ & 6.9989 \\
\hline
\end{tabular}

La figura 5 muestra el mecanismo actuador con las dimensiones obtenidas en las simulaciones numéricas, así como el diseño en CAD de la falange proximal. 


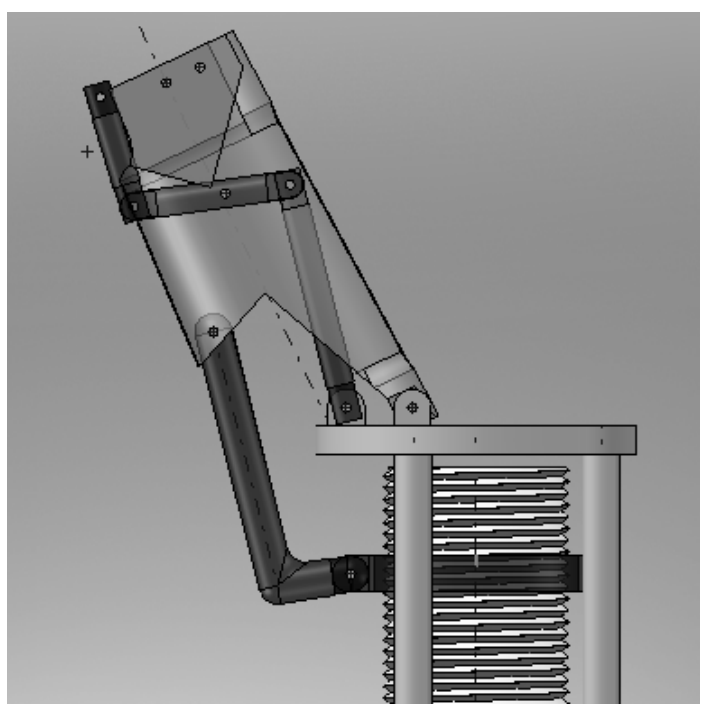

Figura 5. Diseño en CAD de la falange proximal

A partir de los datos de la tabla 3, se calcularon las posiciones de cada uno de los eslabones del mecanismo de cuatro barras. Dichos valores se muestran en la tabla 5.

Tabla 5. Posiciones de los eslabones del mecanismo

\begin{tabular}{|c|c|c|c|c|c|c|c|}
\hline \multicolumn{2}{|c|}{ coordenadas I } & \multicolumn{2}{c|}{ coordenadas $\mathrm{C}$} & \multicolumn{2}{c|}{ posición $\mathrm{H}$} & \multicolumn{2}{c|}{ posición D } \\
\hline $\mathrm{X}$ & $\mathrm{Y}$ & $\mathrm{X}$ & $\mathrm{Y}$ & $\mathrm{X}$ & $\mathrm{Y}$ & $\mathrm{X}$ & $\mathrm{Y}$ \\
\hline 10.3714 & 17.6761 & 15.4969 & 26.4008 & -6.8999 & 0.0000 & 0.0000 & 0.0000 \\
\hline 6.6795 & 20.6658 & 9.4377 & 29.1219 & -6.8999 & 0.0000 & 0.0000 & 0.0000 \\
\hline 2.4850 & 22.8960 & 0.9120 & 30.5994 & -6.8999 & 0.0000 & 0.0000 & 0.0000 \\
\hline-2.0581 & 24.2850 & -6.1660 & 29.9856 & -6.8999 & 0.0000 & 0.0000 & 0.0000 \\
\hline-6.7826 & 24.7816 & -13.1970 & 27.6223 & -6.8999 & 0.0000 & 0.0000 & 0.0000 \\
\hline-11.5152 & 24.3675 & -18.5193 & 24.3760 & -6.8999 & 0.0000 & 0.0000 & 0.0000 \\
\hline-16.0817 & 23.0581 & -22.7672 & 20.4648 & -6.8999 & 0.0000 & 0.0000 & 0.0000 \\
\hline-20.3145 & 20.9013 & -25.7831 & 16.5041 & -6.8999 & 0.0000 & 0.0000 & 0.0000 \\
\hline-24.0580 & 17.9766 & -28.0625 & 12.2333 & -6.8999 & 0.0000 & 0.0000 & 0.0000 \\
\hline-27.1747 & 14.3913 & -29.5936 & 7.8343 & -6.8999 & 0.0000 & 0.0000 & 0.0000 \\
\hline-29.5500 & 10.2771 & -30.4330 & 3.3148 & -6.8999 & 0.0000 & 0.0000 & 0.0000 \\
\hline-31.0967 & 5.7854 & -30.5878 & -1.2428 & -6.8999 & 0.0000 & 0.0000 & 0.0000 \\
\hline-31.7578 & 1.0810 & -30.0724 & -5.7276 & -6.8999 & 0.0000 & 0.0000 & 0.0000 \\
\hline
\end{tabular}


La figura 6 muestra los valores experimentales y obtenidos mediante simulación numérica de las diez posiciones del mecanismo actuador. Como puede observarse, el error de posición para cada uno de los diez puntos de precisión, es despreciable.

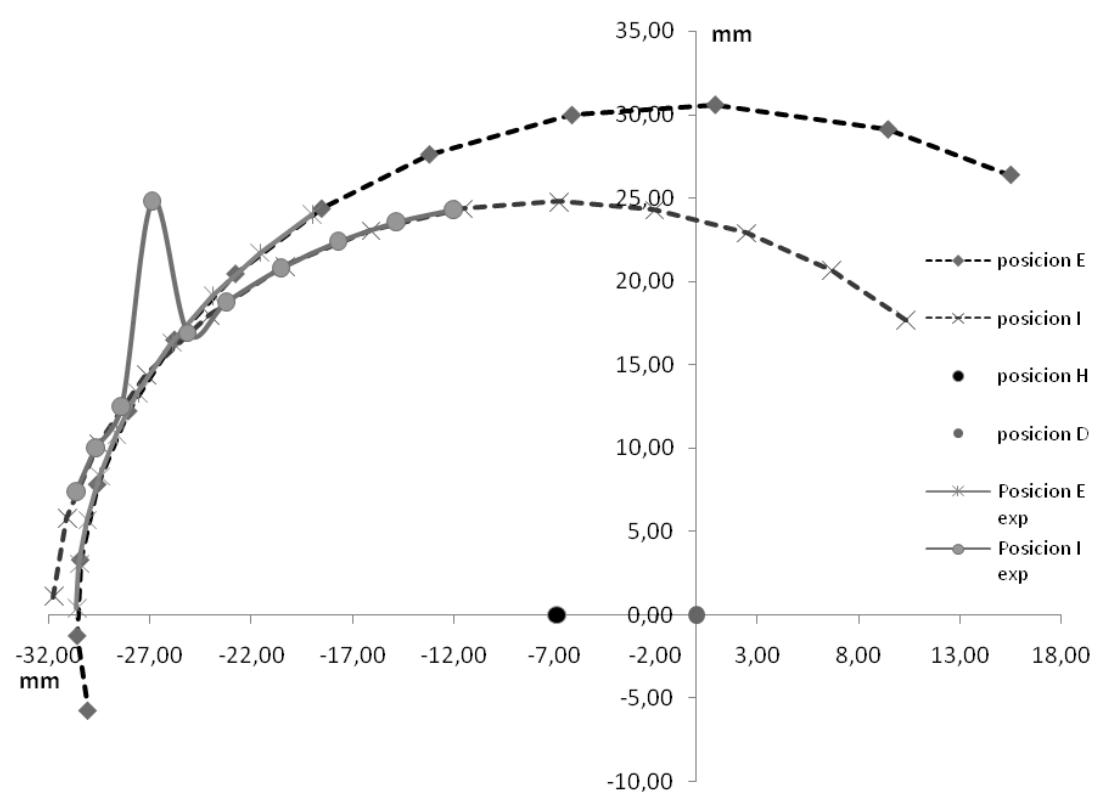

Figura 6. Gráfico de los resultados teóricos y experimentales

\section{CONCLUSIONES}

En el artículo, se presenta la metodología para diseñar un mecanismo de cuatro barras que permite simular el movimiento del dedo humano, y que utiliza un único actuador. Se presenta sólo el desarrollo para la falange proximal ya que para las falanges media y distal, el análisis es similar. Si bien Aviles [11], presenta el diseño y la construcción de un dedo antropomórfico, allí no se muestra una metodología de diseño paramétrico, pero sí un análisis gráfico.

Se ha presentado el análisis de posición de un mecanismo cruzado de cuatro barras, con el cual se obtuvo una descripción matemática para su dimensionamiento físico. Lo anterior se logra mediante un modelo parametrizado del mecanismo en estudio, el cual se resolvió, utilizando herramientas de análisis numérico de $M A T L A B^{\circledR}$. De las soluciones obtenidas, se construyó el mecanismo actuador y el dedo robot antropomórfico mediante herramientas de CAD. Con dicho modelo en CAD, se comprobó la cinemática del mecanismo y la factibilidad de construcción del prototipo, lo cual establece que la metodología de análisis utilizada puede ser viable para mecanismos equivalentes. 
La importancia de este trabajo es la propuesta de una metodología de diseño que describa paramétricamente un dedo mecánico de características antropomórficas, lo cual permite el rediseño del mismo, considerando diferentes valores de entrada, los cuales son establecidos por las necesidades de diseño, sin que se deba llevar a cabo experimentalmente, nuevas mediciones.

Este trabajo es una primera parte de una metodología de diseño concurrente que considera el diseño de mecanismos, bajo un concepto de diseño integrado, en el cual los aspectos mecánicos, electrónicos y de control se consideran en forma simultánea.

\section{REFERENCIAS BIBLIOGRÁFICAS}

[1] Quiroz Gutiérrez, F. (1962) “Anatomía humana”, Ed. Porrúa México, pp. 264-277.

[2] Mason Matthew T., Salisbury Jr J. Kenneth. (1985) "Robot hands and the mechanics of manipulation". The MIT Press Series in Artificial Intelligence.

[3] Jacobsen S. C., Wood J. E., Knutti D. F., and Biggers K. B. (1986) "The UTAH/MIT dextrous hand: Work in progress". In Robot, Grippers, Springer-Verlag, Berlin.

[4] Carrozza M. C., Suppo C., Sebastiani F., Massa B., Vecchi F., Lazzarini R., Cutkosky M. R. and Dario P. (2004) "The SPRING hand: development of a self-adaptive Prosthesisfor Restor- ing Natural Grasping”, Autonomous Robots, vol. 16, $\mathrm{n}^{\circ} .2$, disponible en: http://www.springerlink.com/content/m3023725t7518203/.

[5] Butterfass J., Hirzinger G., Knoch S., and Liu H. (1998) 'DLR's multisensory articulated hand" In Proc. of the 1998 IEEE Int. Conf. on Robotics and Automation, Leuven, Belgica.

[6] Avilés Sánchez O. F., Leon Simanca P. y Calle T. G., (2005) "Dedos para grippers robóticos", Revisión Bibliografíca., Scientia et technica, vol. 11, no. 27, pp. 97103.

[7] Ocampo Gustavo A. Rondón Julián, (2004) "Diseño y construcción de una Mano robot de cuatro dedos que imite los modelos prensiles humanos", Universidad Militar Nueva Granada.

[8] Ceccarelli M., Nava Rodríguez N. Eduardo, Carbone G., (2006) "Optimal design of driving mechanism in A 1-D.O.F. Anthropomorphic finger"., Mechanism and machine theory, vol. 41, no. 8, pp. 897-911. 
[9] García F., Martínez Alvarez J., Saltarén Pazmiño R. J., Guerrero González A., y López Coronado J. “Diseño mecatrónico de un dedo antropomórfico", Universidad Politécnica de Cartagena / Murcia, España. Disponible en: http://www.ceaifac.es/ actividades/jornadas/XXII/documentos/H_05_R.pdf.

[10] Lotti F., Basura G., "A Novel Approach to Mechanical Design of Articulated Fingers for Robotic Hands". Disponible en: http://www.diem.inibo.it/personale/lotti/Articoli_ UBhandIII\%5Ciros_02PDF

[11] Avilés O. Fernando, Simanca P. L. y Niño P. A., "Diseño y construcción de un dedo para grippers robóticos"., presentado en el Congreso Iberoamericano de Ingeniería Mecánica, Perú, 2007.

[12] Edward Shigley J. y Joseph Uicker J., (1988) “Teoría de Máquinas y Mecanismos”, Mc Graw Hill, México.

[13] Hogart B., (1988) "Drawing dynamic hands", Watson-Guptill publications / New York.

[14] Tubiana R., (1980). "Physiologie des mouvements et prehension", Traire de Chirurgie de la main, Anatomie, Physiologie, Biologie, Ed Massons.

[15] De la Caffiniere J. Y., and Malek R., (1981) "Chirurgie réparatrice du pouce”, Ed. Masson. 
